\title{
Frequency-Dependent Impedance Analysis for Discriminating Cancer and Normal Cells Using Microchamber Array
}

\author{
YoonHee Chang, Jiyoon $\mathrm{Bu}$, and Young-Ho Cho* \\ Department of Bio and Brain Engineering, Korea Advanced Institute of Science and Technology (KAIST), \\ 271 Daehak-ro, Yuseong-gu, Daejeon 34141, Republic of Korea
}

(Received May 13, 2016; accepted October 26, 2016)

Keywords: single cell characterization, cancer cell discrimination, cell impedance analysis

We present an impedance monitoring microchamber array for discriminating normal and cancer cells, the top layer of which is covered to form a stable contact between the cells and the electrodes in each microchamber. Compared with devices in previous works, the present device is capable of maintaining long-term cell-to-electrode contact, simply by loading the cells on the electrodes placed in each microchamber and covering the layer, thereby achieving frequency-dependent impedance analysis without additional apparatus. By comparing the impedance response of two human lung cancer cell lines with a normal lung cell line, we verified that the cancer cells are clearly distinguishable against the normal cells by showing both lower resistance ( $241 \mathrm{vs} 271 \mathrm{k} \Omega$ ) and capacitance ( $3.13 \mathrm{vs} 7.01 \mathrm{nF}$ ) in the frequency range of $200 \mathrm{~Hz}$ to $2 \mathrm{MHz}$. Simplicity of cell loading and the capability for determining characteristics of the cancer cells were demonstrated using the device.

\section{Introduction}

Cellular analysis is an effective tool for investigating various biological activities. ${ }^{(1)}$ Over the past decade, cellular analysis has been developed for a wide range of applications from fundamental biological research to optimal treatment determination. Among a range of applications, several effective applications have been proposed for the accurate diagnosis of various diseases, especially cancer. For cancer diagnosis, discrimination between cancer cells and normal cells is essential.(2) Complicated genetic or protein analysis was previously indispensable for determining whether a cell was cancerous or not. However, these methods strongly depended on fluorescent or radio labelling, which necessarily involves the destruction of cells. Thus, the labelling processes associated with these methods leads to a loss of very important biological information which is inherent in live cells.

Recently, label-free and non-invasive methods for detecting and analysing cellular properties have been proposed. Among a number of non-invasive methods for cellular analysis, electrical impedance spectroscopy (EIS), which monitors and analyses the electrical properties of live cells in the frequency domain, has received considerable attention due to its simplicity of use. EIS provides electrical information on cellular properties which is correlated with electrophysiological states, plasma membrane structure, and intracellular contents of cells. It has been established that 
abnormal cells, including cells with cancerous characteristics or cells infected by bacteria, show different electrical characteristics compared to normal cells due to alterations in their ion channel activities or cytoplasm contents. ${ }^{(3-9)}$ Due to the cell and mitochondrial membrane degeneration occurring during cancerization, membranes become more permeable, so that various ions can permeate through the membranes in the case of cancerous cells. ${ }^{(10)}$ In addition, cancer cells have different lipid contents compared with normal cells, which also affects their electrical properties. ${ }^{(11)}$ For these reasons, the electrical properties of cells may be a key markers for the early detection of cancer.

Based on the EIS technique, many researches have worked to classify and detect cancer cells. The conventional impedance-based cell analysis chip can be categorized into two subgroups: the impedance analysis chip for moving cells, and that for captured cells. The previous flowing-cell analysis chips using microchannels ${ }^{(12)}$ were convenient for measuring electrical signals since they do not require additional cell capturing structures. However, owing to flow control, they have difficulties achieving single-cell loading. In addition, these methods do not allow enough time for cell-to-electrode contact to monitor accurate frequency-dependent impedance responses. Therefore, a large number of repeated experiments is required to obtain a frequency-dependent response, since electrical properties for a certain frequency level must be obtained one by one. To overcome these problems, cell impedance analysis chips clamped by membranes with applied external pressures, cell capturing structures, or dielectrophoresis (DEP) forces have been proposed. ${ }^{(13,14)}$ To form a contact between cells and electrodes, pneumatic membranes were inserted, supplemented pillar structures were formed, or additional electric signals were applied. These methods, however, require complex sample controls to position cells on electrodes, and precise contact between cells and electrodes is still difficult to achieve.

In this study, we designed, fabricated, and characterized a simple single-cell impedance analysis chip using an SU8 microchamber array which is capable of forming a stable cell-to-electrode contact in each microchamber. Unlike previous single-cell impedance analysis devices, this chip simply maintains sufficient cell-to-electrode contact time by locating single cells on electrodes placed in each microchamber by covering the top polydimethylsiloxane (PDMS) layer, thus achieving frequency-dependent single cell impedance analysis without additional apparatus or complex flow control. This impedance analysis chip is not only capable of discriminating cells based on their electrical properties but also measuring single-cell electrical properties for advanced cellular analysis, for example, cancer diagnosis.

\section{Materials and Methods}

\subsection{Working principles}

The microchamber array described herein (Fig. 1) consists of a 24-chamber array with electrodes fabricated on the glass substrate for single cell positioning and the top PDMS cover for the cell-to-electrode contact. We seeded a single cell in each microchamber by pouring in the cell mixtures and then placed the PDMS cover on the microchamber array [Fig. 1(c)] to make stable cell-to-electrode contacts. Then we measured the frequency-dependent impedance signal using an impedance analyser. 

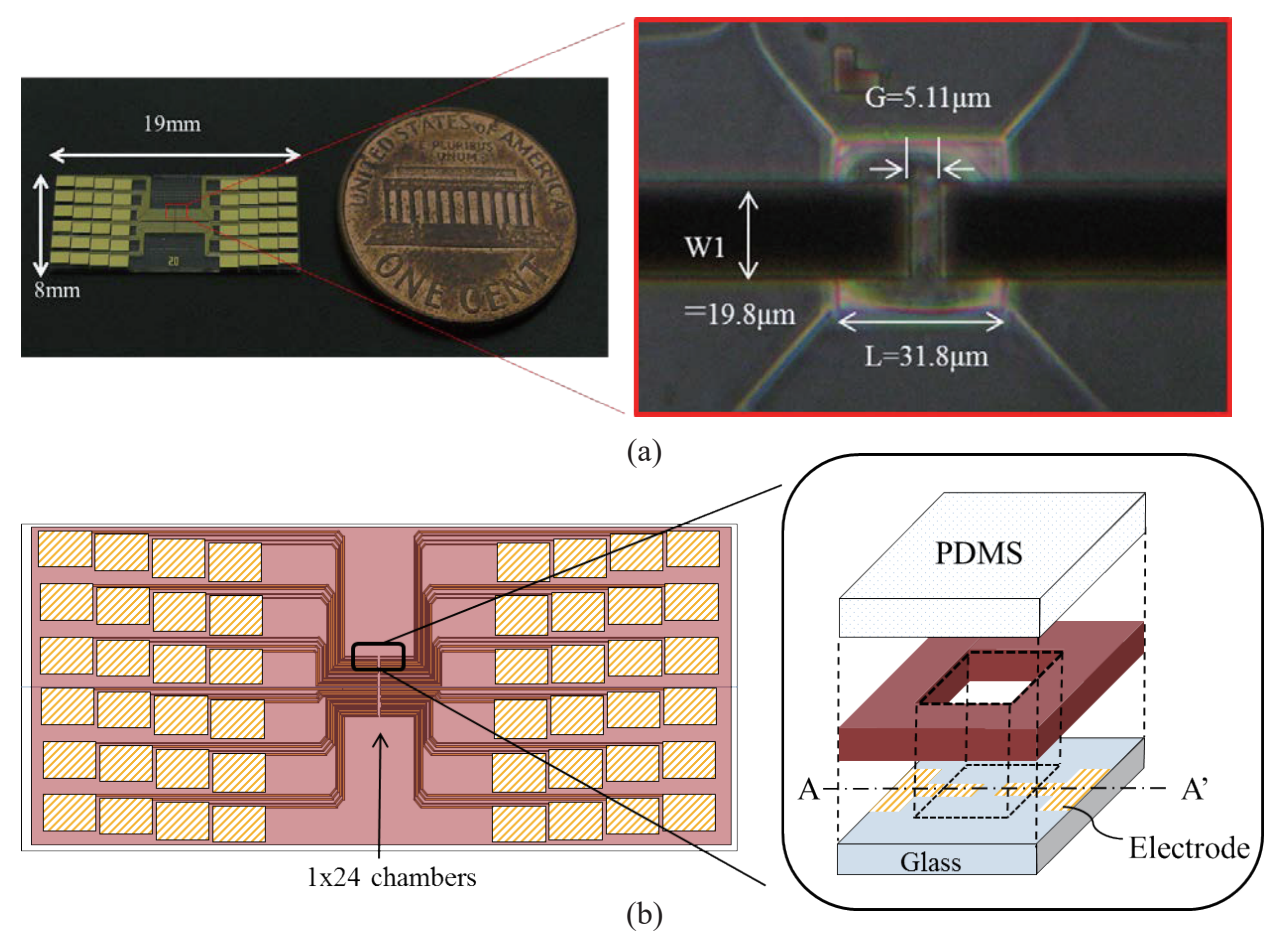

(b)
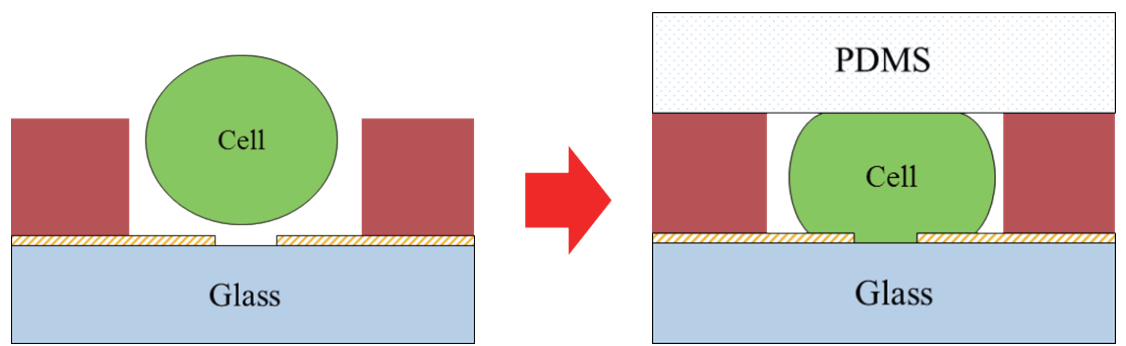

(c)

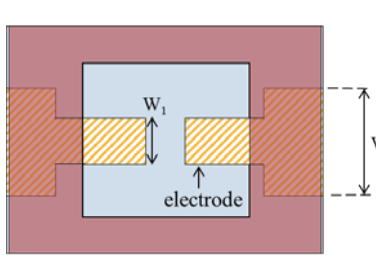

\begin{tabular}{|c|c|c|}
\hline \multicolumn{2}{|c|}{ Parts } & Dimensions $[\boldsymbol{\mu m}]$ \\
\hline Chamber width & $\boldsymbol{L}$ & 32.0 \\
\hline Chamber height & $\boldsymbol{H}_{\boldsymbol{c}}$ & 12.0 \\
\hline Electrode gap & $\boldsymbol{G}$ & 5.00 \\
\hline \multirow{2}{*}{ Electrode width } & $\mathbf{W}_{\mathbf{1}}$ & 20 \\
\cline { 2 - 3 } & $\mathrm{W}_{\mathbf{2}}$ & 40 \\
\hline \multirow{2}{*}{ Electrode height } & $\boldsymbol{H}_{\boldsymbol{e}}$ & $1650(\AA)$ \\
\hline
\end{tabular}

(d)

Fig. 1. (Color online) Single cell impedance analysis chip: (a) Fabricated device; (b) schematic view; and (c) impedance measurement procedures; and (d) top view of a single chamber.

\subsection{Design}

The volume of each microchamber was $32 \times 32 \times 12 \mu \mathrm{m}^{3}$, so that the PDMS cover could trap an isolated single cell. The diameter of the cell-to-electrode contact area was $80 \%$ of the diameter of a cell (approximately $20 \mu \mathrm{m}$ ). The electrode width was $20 \mu \mathrm{m}$, considering the case when the array 
is mounted on the microscope. The gap between the electrodes was $5 \mu \mathrm{m}$ to take account of signal analysis and fabrication error. The exposed area of each electrode in a single chamber was $20 \mu \mathrm{m}$ wide and $13.5 \mu \mathrm{m}$ long. The top view of a single chamber and dimensions are shown in Fig. 1(d).

\subsection{Fabrication}

The fabrication process of the microchamber array is carried out in two steps: fabrication of the bottom array for impedance analysis, and fabrication of the PDMS cover (Fig. 2). The bottom array and the PDMS cover were assembled using a jig after fabrication. A Cr/Au layers, 150 and $1500 \AA$ thick, respectively, were evaporated on a $400 \mu \mathrm{m}$ thick pyrex glass wafer, and electrodes for the impedance analysis were patterned. The electrodes were made by AZ1512 photoresist patterning and $\mathrm{Cr} / \mathrm{Au}$ etching. Then, the $12 \mu \mathrm{m}$ thick SU-8 photoresist was coated and patterned to form a bottom array. The PDMS cover was fabricated using a PDMS micromolding technique. A prepolymer mixture was made with a ratio of curing agent to PDMS (Sylgard 184, Midland, MI, USA) of 1:10 (wt) to make a PDMS cover. The PDMS prepolymer mixture was poured onto the mold and degassed in a vacuum chamber to prevent the formation of bubbles in the fabricated PDMS cover structures. After curing the PDMS mixture for $2 \mathrm{~h}$ at $65^{\circ} \mathrm{C}$, the PDMS replica was peeled from the mold. The PDMS replica was cut and put in a jig for assembly. The jig was madeto-order.

\subsection{Cell preparation}

We used human lung cancer cells (H358, A549) and normal lung cells (Human brachial/tracheal epithelial cells, NHBE). The human lung cancer cells were cultured in cell culture media consisting of RPMI (Gibco) supplemented with 10\% (v/v) fetal bovine serum (FBS, Gibco), and 1\% (v/v) penicillin-streptomycin (Invitrogen). The normal cells were maintained in cell culture media (BEBM).

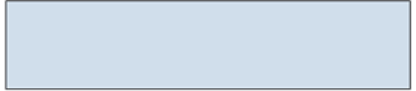

(a) Wafer preparation (Pyrex glass wafer, $500 \mu \mathrm{m})$

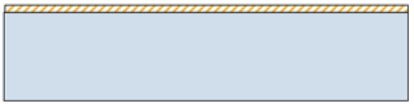

(b) $\mathrm{Cr} / \mathrm{Au}$ deposition $(150 \AA / 1500 \AA)$

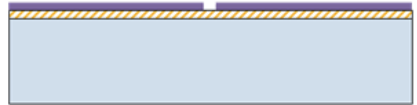

(c) PR patterning (AZ1512, $1.2 \mu \mathrm{m})$

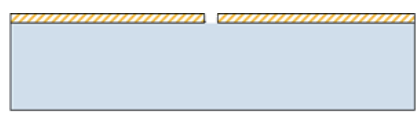

(d) $\mathrm{Cr} / \mathrm{Au}$ etching \& $\mathrm{PR}$ removal

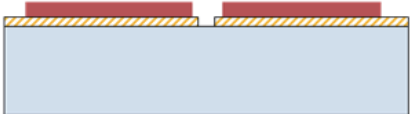

(e) PR patterning (SU-8 2010, 12 $\mu \mathrm{m})$

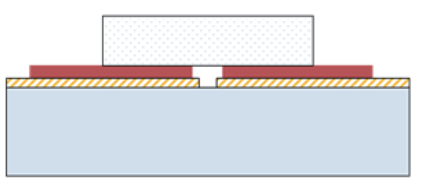

(f) Combined PDMS cover peeled off from Si wafer

Fig. 2. (Color online) Fabrication procedure of the impedance analysis chip. 
The cells were cultured in a $\mathrm{CO}_{2}$ incubator (Sanyo, MCO-18AIC) in an environment at $37{ }^{\circ} \mathrm{C}$ and with $\mathrm{CO}_{2}$ levels of $5 \%$. We suspended the cells in Dulbecco's phosphate buffered saline (PBS) at concentrations ranging from $1.0 \times 10^{6}$ cells $/ \mathrm{ml}$ to $5.0 \times 10^{6}$ cells $/ \mathrm{ml}$, increasing by $0.5 \times 10^{6}$ cells/ $\mathrm{ml}$. The optimal concentration was $3.0 \times 10^{6} \mathrm{cell} / \mathrm{s} / \mathrm{ml}$ and the efficiency for single cell positioning in each microchamber was $29.2 \%$.

\subsection{Cell loading}

Cells were seeded into the microchamber array by simply pipetting $2 \mu \mathrm{l}$ of PBS solution containing a certain number of cells. The numbers of chambers occupied with single cells were counted by microscopy after cell loading with various cell concentrations, starting from $1.0 \times 10^{6}$ cells $/ \mathrm{ml}$ to $5.0 \times 10^{6} \mathrm{cells} / \mathrm{ml}$ and increasing by $0.5 \times 10^{6} \mathrm{cells} / \mathrm{ml}$ in a stepwise fashion.

\subsection{Cell-to-electrode contact verification}

The cell-to-electrode stability was confirmed by the contact rate, the coefficient of variations (CV), and the contact area rate. ${ }^{(15)}$ Each term is defined as follows. High contact rate, high contact area rate, and low $\mathrm{CV}$, are desired to achieve intimate contact between cells and electrodes.

$$
\begin{gathered}
\text { Contact rate }=\frac{\text { No. of cells contacted to both electrodes }}{\text { No.of cells loaded in each chamber }} \times 100(\%) \\
\text { Contact area rate }=\frac{\text { Cell-to-electrode contact area }}{\text { Total cross-sectional area of cell }} \times 100(\%) \\
C V=\frac{\text { Standard deviation of contact area rate }}{\text { Mean value of contact area rate }}
\end{gathered}
$$

\subsection{Cell impedance analysis}

Discrimination between cancer and normal cells by the impedance analysis chip was performed using an LCR meter (Agilent, E4980A). The magnitude and the phase of the impedance signal was measured in the frequency range of $200 \mathrm{~Hz}-2 \mathrm{MHz}^{(16)}$ with $100 \mathrm{mV}_{\mathrm{p}-\mathrm{p}} \mathrm{AC}$ signals. Figure 3 indicates the equivalent electrical circuit model ${ }^{(13)}$ for cell impedance analysis. The cell impedance, $Z_{\text {cell }}$, consists of the cell capacitance $\left(C_{c}\right)$ and resistance $\left(R_{c}\right) . C_{d i}$ indicates the dielectric capacitance between the electrode and cell, which includes various capacitances that are derived from two electrodes. $R_{e}$ indicates the electrode resistance. Based on the electrical equivalent circuit model, we verified the electrical properties of normal cells and cancer cells. The electrical properties of the equivalent circuit model (Fig. 3) were calculated and the values of $R_{e}$ and $C_{d i}$ were extracted by curve fitting for the cells. 


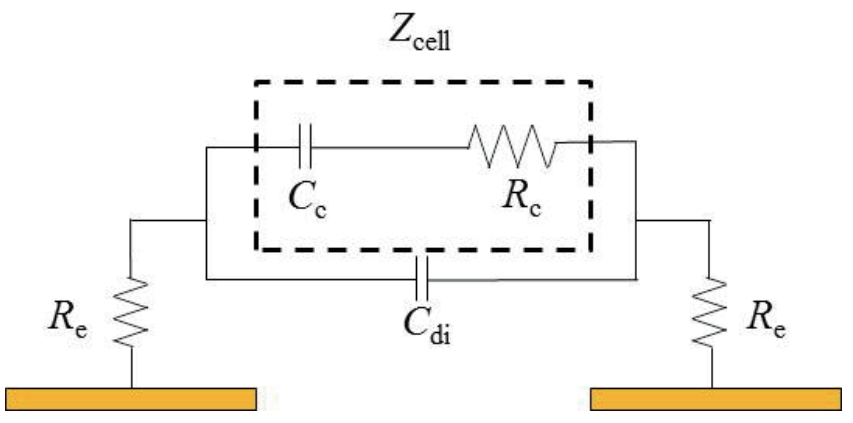

Fig. 3. (Color online) The equivalent electrical circuit model.

\section{Results and Discussion}

\subsection{Single cell loading}

Before measuring the cell-to-electrode contact stability, we measured the diameter of the cell size for each cell line, to confirm that our chip design was appropriate for single-cell loading. The average diameter of A549, H358, and NHBE cell lines were $19.5 \pm 1.2,21.1 \pm 1.1$, and 20.2 $\pm 1.3 \mu \mathrm{m}$, respectively. Therefore, the width of a single chamber was 1.57 times longer than the average diameter of the cells, which is in the range of the optimal condition for singlecell loading. ${ }^{(17)}$ Figure 4 shows the number of chambers occupied with respect to the cell concentrations. The number of chambers occupied by single cells shows an increasing tendency as cell concentration increases. The largest and the second largest occupancy are shown when the cell concentration was $5.0 \times 10^{6}$ and $3.0 \times 10^{6} \mathrm{cells} / \mathrm{ml}$, respectively. However, we determined the optimized cell concentration to be $3.0 \times 10^{6} \mathrm{cell} / \mathrm{s} / \mathrm{ml}$, since a large amount of cell loss occurred when 5.0 $\times 10^{6} \mathrm{cells} / \mathrm{ml}$ were loaded.

\subsection{Cell-to-electrode contact stability verification}

The experimental results to confirm cell-to-electrode stability are shown in Table 1. The contact rates, which are defined as the number of cells contacting both electrodes with respect to the total number of single cells that have been trapped, were $94.44 \pm 3.92,94.44 \pm 3.92$, and $97.22 \pm 3.92 \%$ for NHBE, A549, and H358 cells, respectively. Therefore, we confirmed that most of the cells allocated in the chamber contacted both electrodes, regardless of the cell type. The contact area rates, which refer to the cell-to-electrode contact area compared to the total cross-sectional area occupied by the cell, were measured as $53.44 \pm 5.37,52.70 \pm 5.21$, and $53.50 \pm 5.24 \%$ for NHBE, A549, and $\mathrm{H} 358$ cells, respectively. The variance in the contact area rate was small, since CV levels were $0.100,0.099$, and 0.098 for each cell line (stable in statistics if under 0.33 ). The results show that more than half of the total area of the cell is in contact with the electrodes, which is sufficient to measure an electrical signal. Therefore, we confirmed that the impedance analysis chip makes stable cell-to-electrode contact. All experiments to demonstrate cell-to-electrode stability have been repeated 12 times. 


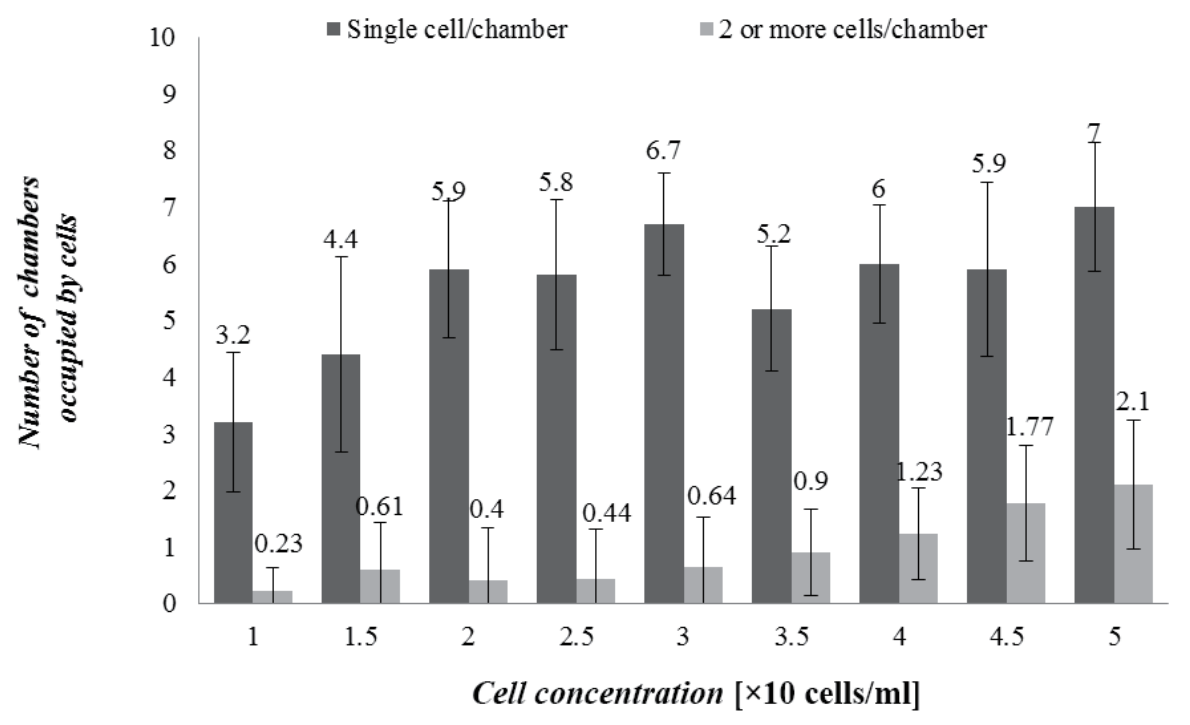

Fig. 4. The number of chambers occupied by a single H358 cell at different cell concentrations.

Table 1

Cell-to-electrode contact rate and contact area rate with coefficients of variation.

\begin{tabular}{lccc}
\hline Cells & Contact rate $(\%)$ & Contact area rate $(\%)$ & Coefficient of variation \\
\hline NHBE & $94.44 \pm 3.92$ & $53.44 \pm 5.37$ & 0.100 \\
A549 & $94.44 \pm 3.92$ & $52.70 \pm 5.21$ & 0.099 \\
H358 & $97.22 \pm 3.92$ & $53.50 \pm 5.24$ & 0.098 \\
Average & $95.37 \pm 4.14$ & $53.21 \pm 5.27$ & 0.099 \\
\hline
\end{tabular}

\subsection{Cell impedance analysis and discrimination of cancer cells}

Under the optimized conditions described, we measured and compared the impedance (magnitude and phase) response of human lung cancer cells (H358, A549) with the response of normal lung cells (the human brachial/tracheal epithelial cells of NHBE) in the frequency range of $200 \mathrm{~Hz}$ to $2 \mathrm{MHz}$ for an AC signal of $100 \mathrm{mV}_{\mathrm{p}-\mathrm{p}}$. Figure 5 shows the impedance magnitude and phase for four different conditions (impedance response of NHBE, A549, H358, and PBS buffer). In both magnitude and phase response, significant differences were observed between normal cells and cancer cells. NHBE cells showed a higher impedance magnitude $(60.07-154.36 \mathrm{k} \Omega$ ) than human lung cancer cells (H358 and A549) in the frequency range from $95.6 \mathrm{kHz}$ to $2 \mathrm{MHz}$, while also showing a higherimpedance phase $\left(3.96-20.80^{\circ}\right)$ than human lung cancer cells in the frequency range from $4.37 \mathrm{kHz}$ to $2 \mathrm{MHz}$. The differences in impedance response are presumably caused by their cellular factors like physiological states, membrane property, and cytosolic characteristics. ${ }^{(3-9)}$ When a single cell is placed inside an electric field, local distortion of the field leads to measurement differences in the 


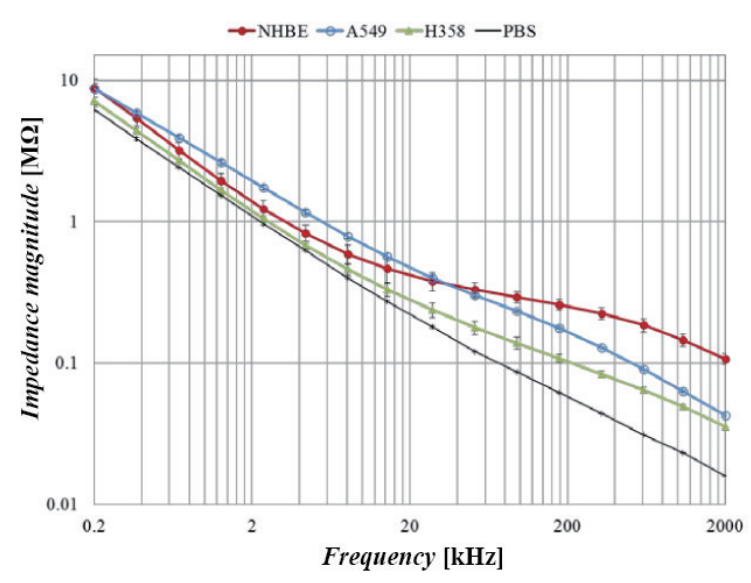

(a)

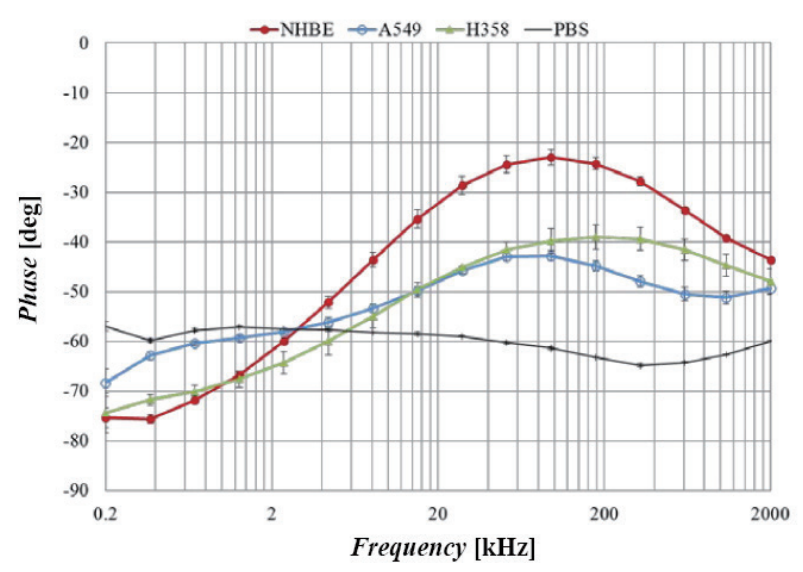

(b)

Fig. 5. (Color online) Impedance response of NHBE, A549, H358, and PBS buffer in the frequency range from $200 \mathrm{~Hz}$ to $2.0 \mathrm{MHz}$ for an $\mathrm{AC}$ signal of $100 \mathrm{mV}_{\mathrm{p}-\mathrm{p}}$ : (a) magnitude and (b) phase angle.

characteristic impedance signature of the cell. This allows for detection of abnormal cells as well as for the classification of normal cell types. ${ }^{(18)}$ The resistance and capacitance of the normal cell are $271 \mathrm{k} \Omega$ and $7.01 \mathrm{nF}$, respectively, which is clearly different from the resistance and capacitance of cancer cells ( $226 \mathrm{k} \Omega$ and $3.30 \mathrm{nF}$ for A549 and $255 \mathrm{k} \Omega$ and $2.95 \mathrm{nF}$ for H358). The average resistance and capacitance of the cancer cells were calculated as $241 \mathrm{k} \Omega$ and $3.13 \mathrm{nF}$, respectively. We confirmed that the electrical parameter values of the H358 and A549 cancer cells are included in the range of those of malignant tumour cells. ${ }^{(13)}$

\section{Conclusion}

This paper presents a simple impedance measuring microchamber arrayon which a PDMS cover makes stable cell-to-electrode contacts in each microchamber and enables the characterization of the frequency-dependent single cell impedance analysis. In the experimental study, we verified single-cell trapping and contact stability by loading varying concentration of cells and calculating cell-to-electrode contact rates. For the impedance analysis, NHBE cells showed a higher impedance magnitude (60.07-154.36 k $\Omega$ ) than human lung cancer cells (H358 and A549) in the frequency range from $95.6 \mathrm{kHz}$ to $2 \mathrm{MHz}$, while showing a higher impedance phase (3.96-20.80 $)$ than human lung cancer cells in the frequency range from $4.37 \mathrm{kHz}$ to $2 \mathrm{MHz}$. As a result, cancer cells showed lower resistance and capacitance $(241 \mathrm{k} \Omega$ and $3.13 \mathrm{nF})$ compared to normal lung cells, NHBE cell lines $(271 \mathrm{k} \Omega$ and $7.01 \mathrm{nF})$. We demonstrated simple single-cell loading and stable cellto-electrode contact capabilities of our microchamber array through experiments discriminating normal cells and cancer cells.

\section{Acknowledgements}

This research was supported by the Converging Research Center Program funded by the Ministry of Science, ICT and Future Planning (Project No. 2015054201). 


\section{References}

1 M. G. Roper: Anal. Chem. 88 (2016) 381.

2 L. Yang: ECS Trans. 50 (2012) 101.

3 D. P. Poenar, C. Iliescu, M. Carp, A. J. Pang, and K. J. Leck: Sens. Actuator, A 139 (2007) 162.

4 S. B. Prakash and P. Abshire: IEEE Sens. J. 7 (2007) 440.

5 S. Gawad, K. Cheung, U. Seger, A. Bertsch, and P. Renaud: Lab Chip 4 (2004) 241.

6 Y. Huang, N. S. Sekhon, J. Borninski, N. Chen, and B. Rubinsky: Sens. Actuator, A 105 (2003) 31.

7 R. Gomez, R. Bashir, A. Sarikaya, M. R. Ladisch, J. Sturgis, J. P. Robinson, T. Geng, A. K. Bhunia, H. L. Apple, and S. Wereley: Biomed. Microdevices 3 (2001) 201.

8 A. Han and A. B. Frazier: Lab Chip 6 (2006) 1412.

9 Y. Park, H. W. Kim, J. Yun, S. Seo, C. J. Park, J. Z. Lee, and J. H. Lee: Biomed Res. Int. (2016). doi: Artn 874802310.1155/2016/8748023

10 P. G. Seeger and S. Wolz: Successful Biological Control of Cancer: By Combat Against the Causes (Neuwieder Verlagsgesellschaft mbH, Gesamtherstellung 1990).

11 E. Revici: Research in Pathophysiology as Basis for Guided Chemotherapy, with Special Application to Cancer (D. Van Nostrand Company, New Jersey, 1961).

12 G. M. Dittami, H. E. Ayliffe, C. S. King, and R. D. Rabbitt: J. Microelectromech. Syst. 17 (2008) 850.

13 L. S. Jang and M. H. Wang: Biomed. Microdevices 9 (2007) 737.

14 G. Kang, Y. J. Kim, H. S. Moon, J. W. Lee, T. K. Yoo, K. Park, and J. H. Lee: Biomicrofluidics 7 (2013).

15 L. S. Jang and M. H. Wang: Biomed. Microdevices 9 (2007) 737.

16 H. P. Schwan: Med. Prog. Technol. 19 (1993) 163.

17 W. C. Lee, S. Rigante, A. P. Pisano, and F. A. Kuypers: Lab Chip 10 (2010) 2952.

18 S. Gawad, L. Schild, and P. Renaud: Lab Chip 1 (2001) 76. 